\section{Las ciencias sociales y la salud en la modernización de Venezuela}

\author{
Social sciences and health in the \\ Venezuelan modernization process
}

Roberto Briceño-León 1

Bailde García 2

Virginia Rodríguez 1

Lya Tovar 3
1 Laboratorio de Ciencias Sociales de la Universidad Central de Venezuela. A partado 47.795, 1041-A, Caracas Venezuela. rbriceno@reacciun.ve 2 Instituto de Biomedicina, Caracas, Venezuela. 3 Decanato de la Facultad de M edicina. Escuela de Salud Pública, Facultad de M edicina de la Universidad Central de Venezuela.
Abstract The social sciences have been involved in health programs in Venezuela since theturn of the 20th Century, but their role has been changing as the country has undergone successive transformations and the scientific community has matured. This article analyzes the social sciences' actions in the medicine/ health field in Venezuela over the course of the 20 th Century. V ariables relating to the changes and/or transformations occurring in society, the State, and academia were specified, permitting the identification of five phases denoting different styles and ways of conceiving the relationship between the social sciences and health: a) the social dimension as a field of medical discovery; $b$ ) the social dimension and environmental health as supports for modernization; c) professionalization of the social dimension - from social medicine to the social sciences; d) the social dimension as a critique of the dominant powers; and e) the social dimension's diversity - from medicine to health. The social sciences' critical and constructiveactions in the field of medicine and health, their contribution to the State's health programs and actions, and research activities have yielded them acknowledgment as a key element of the social changes required for the so-far inconclusive modernization of Venezuelan society. Key words Social sciences, $\mathrm{H}$ ealth, Venezuela, Social Change, Medicine
Resumen Las ciencias sociales han estado vinculadas a los programas de salud en Venezuela desde comienzos del siglo 20, pero su papel ha ido cambiando conforme se han dado las transformaciones en el país y en la maduración de la comunidad científica. Esteartículo presenta un análisis sobre la actuación de las ciencias sociales durante el siglo 20 en el campo de la medicina y la salud, en Venezuela. Se establecieron variables relacionadas con los cambios y/o transformaciones que se han dado en la sociedad, el Estado y la academia, las cuales permitieron construir cinco fases que denotan los estilos y formas de concebir la relación entre las ciencias sociales y la salud: a) lo social como un asunto de descubrimiento médico; b) lo social y lo sanitario como apoyo a la modernización; c) la profesionalización de lo social: de la medicina social a las ciencias sociales; d) lo social como crítica al poder; e) la diversidad de lo social: de la medicina a la salud. La actuación crítica y constructiva de las ciencias sociales en el campo de la medicina y la salud, su contribución en los programas y acciones en salud desarrolladas por el Estado y, en el área de la investigación, le han otorgado el reconocimiento como claves en los cambios sociales necesarios para la modernización, hasta ahora inconclusa, de la sociedad venezolana. Palabras-claves Ciencias sociales, Salud, Venezuela, Cambios sociales, M edicina 


\section{Introducción}

Las ciencias sociales han estado vinculadas a los programas de salud en Venezuela desde comienzos del siglo 20, pero su papel ha ido cambiando conforme se han dado las transformaciones en el país y en la maduración de la comunidad científica. En este trabajo nos proponemos describir esas mutaciones y ofrecer una interpretación de dichos cambios de acuerdo a cuatro variables que consideramos fundamentales desde la perspectiva de la sociología de la ciencia para poder entender el proceso de constitución y cambio de una comunidad científica y de un campo del conocimiento y de la práctica profesional.

Las cuatro variables sobre las cuales será desarrollado este análisis son:

1) Los cambios sociales que ocurren en el país y que están relacionados a partir de 1930 por la presencia de las actividades de exploración y explotación del petróleo. Se trata de observar cómo los cambios en la sociedad se reflejan en las condiciones de salud de la población y en consecuencia en la manera cómo las ciencias sociales se incorporan en su interpretación y modificación.

2) Las transformaciones que ocurren en el aparato del Estado, en particular en las instituciones del sector salud y en las políticas de salud y que son el producto tanto de los cambios en la economía del país como en las concepciones acerca del papel del Estado en la sociedad.

3) La evolución del desarrollo académico y profesional de las ciencias sociales, es decir, de la existencia de estudios profesionales de pregrado y postgrado que formen a los individuos y de centros de investigación que pueden contribuir a la expansión del campo del conocimiento.

4) Las teorías dominantes en el pensamiento científico, tanto por su imposición como paradigma en un momento dado, como con las modas intelectuales que colocan en la cima de la popularidad a unos autores o corrientes en detrimento de otras.

Con estos cuatro componentes hemos podido establecer las fases de presencia de las ciencias sociales en la medicina y la salud en Venezuela. Estas fases tienden coincidir con los años, pero no siempre es así, pues en al gunos casos, se superponen y aparece una nueva tendencia cuando aun la otra no ha desaparecido completamente, por lo tanto no debemos tomarlos como períodos históricos propiamente dichos sino como unos estilos y formas de con- cebir la relación entre las ciencias sociales y la salud en un tiempo histórico difuso.

\section{Fase I - Lo social como asunto Descubrimiento médico}

Lo social ingresa al mundo de la medicina y la salud de la mano de los médicos que tenían sensibilidad social y de aquellos que, interesados en la salud pública, sentían la obligación de comprender las causas de la morbilidad y mortalidad de la población más allá de la simple causa inmediata. Es así como es posible encontrar descripciones de la situación social por parte de algunos médicos quienes procuran identificar en las condiciones materiales de vida o en los hábitos individuales los factores que inducen a la aparición de alguna enfermedad. El componente de aplicación de estos conocimientos tenía su contraparte en las actividades de asistencia social que debían emprender unos auxiliares que constituían una suerte de enfermeros desde el punto de vista social, eran auxiliares de la labor médica.

Durante los primeros 30 años del siglo 20, Venezuela era fundamentalmente una sociedad rural que producía café y cacao en condiciones semi-feudales y las exportaba al mundo capitalista. Poco más del $80 \%$ de la población vivía en las zonas rurales con una el evada mortalidad infantil y cuya población estaba sometida a una alta morbilidad y mortalidad de malaria y tuberculosis. Esta situación comienza a cambiar a partir de 1926 cuando el valor de las exportaciones de petróleo superan por primera vez lo que el país recibía por el café, que para ese entonces era el principal rubro de exportación. A partir de allí se fortalece el poder del gobierno central, pues la crisis capitalista de los años treinta limitaron las exportaciones de productos agrícolas en primer momento y, luego, cuando se revaloriza la moneda nacional ante el dólar, se pierde competitividad ante los otros países y se fortalece la dependencia del país del petróleo y se consolida el poder del gobierno central que procede a generar transformaciones importantes en la estructura del Estado.

H asta 1936 existía en el país un M inisterio de Sanidad y Agricultura y Cría, pero en eseaño se separan las funciones de ese ministerio y se crea el M inisterio de Sanidad y Asistencia Social, creándose bajo ese mismo decreto la Dirección de Asistencia Social. Es importante señalar cómo la primera vez que aparece la idea de 
lo social de una manera explícita en los organismos del Estado venezolano, lo hace ligado a la salud de la población, es decir, se considera que la asistencia social formaba parte de los planes de atención a la salud de la población.

En 1937 se creó el curso de médicos higienistas a cargo del ministerio, lo que vendría ser los orígenes de los cursos de salud pública y en lo cuales existía una mayor presencia de lo social a partir de considerar la higiene como un asunto relacionado con las condiciones sociales.

En el año 1938, se crean los Distritos Sanitarios, modelo de organización en salud muy exitoso por varias décadas en el desarrollo de políticas de salud local, e inclusive base de referencia para al gunos países de América Latina y el Caribe.

Con este nuevo modelo de gestión, se da apertura a las U nidades Sanitarias, espacios de salud de atención integral a la población, con énfasis en la prevención, favoreciendo la ampliación del espacio social, específicamente a través de la figura del Servicio Social; siendo su objetivo apoyar los programas de salud pública, a través de actividades de educación sanitaria.

Paralelamente, ante la necesidad de ir tecnificando el personal de trabajadores sociales ubicados en diferentes servicios de salud del países crea, en el año de 1941, la primera Escuela de Servicio Social patrocinada por el M inisterio de Sanidad y Asistencia Social. El objetivo de la formación de la escuela era la de reforzar la metodología de trabajo social de casos, la cual se venía aplicando a nivel de los servicios de salud donde se prestaba atención médica a pacientes afectados por diferentes patologías.

Por otra parte, el Ministerio de Sanidad, en el año de 1946, transforma la sección de Propaganda Sanitaria en la División de Educación Sanitaria, para fortalecer el trabajo sanitario, con base esencial en la orientación de la educación higiénica del público apoyado por el periódico Ser y el periódico mural Pueblo y Salud.

A partir de esta visión sanitaria, el trabajador social articula las actividades de divulgación de los mensajes de prevención y educación a los diferentes públicos/pacientes que acudían a las consultas, e igualmente, apoyar el trabajo de visitas domiciliarias programadas en función del seguimiento de la rehabilitación de las personas egresadas de los centros de atención.

En esta época se inician también las actividades de la psicología, la cual por su carácter individual tenía mayor aceptación; en los años treinta se inicia la aplicación de los "test mentales", siendo uno muy importante la aplicación del test de Binet-Simón para la selección de enfermeros y que fue una importante tesis doctoral en medicina a fines de esa década. Ya en los años cuarenta se difunden estas técnicas de medición y en 1943 se creó la sección de psicometría del Servicio de H igiene Escolar.

Durante esta fase, como habrá podido observarse lo social se refiere exclusivamente a las condiciones individuales o familiares que deben ser modificadas por una intervención que modifique el comportamiento. No hay una visión colectiva ni estructural del proceso saludenfermedad, lo que se debía cambiar eran las personas, no la organización de la sociedad. Y para cambiar las personas se consideraba que era muy importante la educación sanitaria.

Como un mecanismo institucional de ampliar la cobertura de educación sanitaria, se establece, en el año de 1948, la creación de la primera comisión interministerial - M inisterio de Sanidad y M inisterio de Educación. Este trabajo fue liderizado por médicos sanitaristas, enfermeras de salud pública y trabajadores sociales, los cuales para el momento representaban un número reducido. No obstante, aunque la participación fue muy pequeña en comparación a otros profesionales de la salud, la misma significó una oportunidad de proyección de las acciones de lo social en el ámbito de las instituciones educativas.

Los principios de educación sanitaria enmarcados en la política ministerial en ese momento tenían un contenido filosófico muy reduccionista del ser humano y sus dimensiones sociales, culturales, económicas y políticas. La misma se sustentaba en criterios, entre ellos:

- La prevención, promoción, educación y fomento de la salud, enmarcado en el modelo de Leavell y Clark, el cual explica la salud en la visión de la evolución natural de la enfermedad, inscrita y diferenciada en los tres niveles de atención médica, en correspondencia al grado de complejidad de la patología presentada por los pacientes tanto hospitalizados como una vez egresados. En este caso, el papel del trabajador social estaba en darle seguimiento a la rehabilitación social a través de charlas, apoyo socioeconómico.

- Una posición bancaria de la educación, diferenciada entre el sujeto/paciente como receptor pasivo de las acciones y el emisor/profesionales de la salud, dueños del conocimiento absoluto, los cuales de acuerdo a su visión y a sus 
expectativas diseñaban y ejecutaban las acciones de educación sanitaria.

- Utilización de técnicas y recursos, como la revista Ser y otros materiales impresos en forma general izante y masiva, sin considerar su validación y sentido de oportunidad en los posibles beneficiarios.

- La preparación y utilización de las charlas como estrategias de educación sanitaria, con una visión escasa de análisis de contenido de acuerdo a las diferentes audiencias a las cuales se dirigía.

- La institucionalidad de las campañas sanitarias, como vía de divulgación de la ejecución de eventos de salud en las comunidades.

En síntesis, la actuación de las ciencias sociales para ese período se determina por un trabajo social de atención de casos de orientación bené fico/asistencialista, subordinado a las decisiones biomédicas del sistema sanitario, de acuerdo al tipo de emergencias de las patologías específicas del momento, como tuberculosis, lepra, paludismo, entre otros. $Y$ de esta manera, buscar respuestas inmediatas y específicas desde una intervención individual o del grupo familiar, obviando el tipo los factores estructurales condicionantes de cada situación.

La validez y legitimidad de la actuación de las ciencias sociales a nivel del sector salud estaban condicionadas a los resultados de una capacidad resolutiva de esfuerzos institucionales, para viabilizar ayudas socioeconómicas para solventar 0 aliviar en al gún grado, las problemáticas presentadas por los pacientes de escasos recursos económicos referidos por los equipos médicos de diferentes servicios de salud del país.

\section{Fase II - Lo social y lo sanitario como apoyo a la modernización (1945-1960)}

La segunda modalidad de relación entre las ciencias sociales, la medicina y la salud se corresponde con la idea de una sociedad en transición que avanza del atraso al desarrollo, de lo tradicional a lo moderno, de lo rural a lo urbano. En ese proceso de cambio social la salud cumple un papel muy importante al garantizar las condiciones saludables de un territorio donde podrían darse el desarrollo económico, y para que la salud puede garantizarse era necesario hacer cambios en las condiciones ambientales que previnieran la enfermedad y cambios en los individuos para "adaptarlos" a las condiciones higiénicas nuevas y a la mentalidad "moderna" que requería el trabajo y la industria.

Este período corresponde al momento de mayor expansión y crecimiento de la economía venezolana, pues coincidía con lo que H osbawn (1995) ha llamado la "edad de oro", pues, el capitalismo central tenía un notable crecimiento por la reconstrucción de Europa después de la Segunda Guerra M undial y la demanda de energía se hizo muy grande por lo que Venezuela se convirtió en el primer país exportador mundial de petróleo, ya que los Estados U nidos pasaron a ser un importador en lugar de exportador del hidrocarburo. Esta fuente de ingresos y una importante inmigración europea propiciaban la idea de un futuro crecimiento agroindustrial y una modernización de la sociedad venezolana que requería de condiciones sanitarias diferentes que permitieran ese añorado desarrollo.

El esquema que mejor responde a esta orientación lo encabeza el equipo encargado del control de la malaria que encabezado por el $\mathrm{Dr}$. Arnoldo Gabaldon realizan la transformación más importante que ha ocurrido en la salud pública de Venezuela. La dirección de malaria fue creada al mismo tiempo que el ministerio, pero pocos años después empieza a adquirir una independencia de la estructura del ministerio, convirtiéndose en lo que para muchos era un ministerio dentro de otro ministerio. En medio de las restricciones económicas y de materia prima que imponía la Segunda Guerra M undial y en un momento en que las instituciones y las personas tendían a concentrarse en Caracas este equipo logró que se le aprobará la construcción de una sede en una ciudad del interior del país, la cual fue inaugurada en 1943. Para 1944 se inició el primer curso internacional de malaria en el cual se incluían tres grandes bloques del conocimiento: la entomología para conocer el insecto vector de la enfermedad, la clínica médica para su diagnóstico y tratamiento y las condiciones sociales de la población. Con esta orientación, se inició en 1945 la primera aplicación del DDT para usos civiles (antes había sido usado por las tropas de USA en la guerra del pacífico), que en tres años hizo descender notablemente la mortalidad y morbilidad por malaria y llevó a que quince años más tarde fuera declarado un territorio de 408 mil kilómetros como la primera zona donde la malaria había sido erradicada.

Pero al mismo tiempo de la lucha contra la malaria ocurre una acción de control de la en- 
fermedad de Chagas para lo cual se inicia un programa de viviendas y los programas de lucha contra la anqui lostomiasis y la bilharzia con obras de saneamiento ambiental. Pero para todo esto se requería de cambios en el comportamiento de los individuos en la manera de cómo se construía la casa, en la forma como se debía usar la poceta y lavar las manos, en la importancia del uso del calzado. Como en ese entonces no existían profesionales, pues no existían todavía en las universidades las carreras que formaran estos recursos, fue necesario trabajar con los asistentes sociales y con los educadores sanitarios que la misma escuela de malaria se dedicó a formar.

Lo que llama la atención en esta fase es que el énfasis se mueve del tratamiento aislado de casos, hacia el tratamiento de individuos pero en colectividades, es decir, se trataba de lograr un cambio en todos los individuos de una comunidad, para que así pudiera cambiar la situación social. Es posible que esta visión tuviera su origen en la manera como se aplicaba el insecticida para la malaria que implicaba el tratamiento de la vivienda aislada sino de todas las viviendas y la medicación de todos los individuos para poder cortar el ciclo de transmisión del parásito.

Por otra parte el M inisterio de Sanidad y Asistencia Social en concordancia a las gestiones públicas del país, genera movimientos en la estructura y funciones en la dirección de una visión de la salud pública básicamente de atención individual, y de atención al público por la vía de las visitas domiciliarias para el se guimiento de la rehabilitación de los pacientes egresados de diferentes centros hospitalarios.

Desde el año 1951 hasta el año 1957, secambia la denominación y posición organizacional de la Dirección de Asuntos Sociales, en una División dependiente de la Dirección de Salud Pública. Este cambio de posición en la estructura obedece a la apreciación gerencial del papel de las ciencias sociales para ese momento como actores secundarios del sistema de salud.

Los profesionales de las ciencias sociales continúan con la filosofía de la educación sanitaria, información y divulgación del conocimiento sanitario a nivel de las instituciones sanitarias, bajo dirección de los equipos médicos quienes de acuerdo a sus criterios de la salud determinaban el tipo de programas y sus contenidos.

Para este momento las tesis de participación comunitaria en América Latina y el Caribe gi- raban en la idea que la participación era un proceso susceptible de estimularse desde afuera, y que la mayor o menor movilización de la población podría ser manipulada por agentes externos. Este concepto de participación a final de esta década se empieza aplicar en Venezuela, en ocasión del cambio de régimen de una dictadura a una democracia representativa.

El enfoque metodológico dominante se centraba en la llamada animación rural o desarrollo de la comunidad, y su objetivo era la transferencia de nuevas tecnologías para mejorar la calidad de vida de la población.

En este caso, la participación de la gente estaba representada por su capacidad de organizarse y movilizarse en torno a programas organizados y dirigidos por el Estado, bajo el supuesto de que toda la población aceptaría con facilidad las ideas, innovaciones y las prioridades indicadas por los profesionales de la salud.

La gestión sanitaria se apropia de este modelo para la proyección de los programas comunitarios. Se apertura del reclutamiento de profesionales de las diferentes disciplinas de las ciencias sociales, iniciándose el programa denominado Bienestar Comunal, el cual abre alternativas para la construcción de un equipo multidisciplinario en ciencias sociales.

\section{Fase III - La profesionalización de lo social: de la medicina social a las ciencias sociales (1960-1970)}

La tercera fase que queremos destacar se corresponde con el proceso de profesionalización de las ciencias sociales y la expansión del sistema educativo. En este momento se da una importante incorporación de las ciencias sociales tanto a la academia como a la gestión de gobierno nacional o local, pero al mismo tiempo se inicia el proceso por el cual el énfasis de la acción del Estado en salud se desplaza de lo preventivo hacia lo curativo y en consecuencia se substituye el énfasis en el saneamiento ambiental por la proliferación de hospitales.

Venezuela estaba saliendo de una década de dictadura y se abría a una joven democracia que debía encontrar legitimidad en medio de amenazas de los militares que deseaban retornar al poder y de un movimiento guerrillero de inspiración cubana que se estableció en las zonas rurales del país. El momento político difícil tuvo repercusiones en la actividad económica y el gobierno debió por un lado establecer restric- 
ciones, pero por el otro desarrollar una política social agresiva con una reforma agraria, una expansión de la matrícula estudiantil desde nivel primario hasta la universidad y una atención a la salud que fuese visible para las familias y que tuviese repercusión en las zonas rurales, pero también en las urbanas pues ya más de la mitad de la población vivía en ciudades.

A nivel internacional en este período suceden eventos importantes en la evolución de las políticas de salud en la región de las Américas, como la reunión de Punta del Este (1961), donde se inicia una era de la promoción concertada del desarrollo social y económico de las Américas, instituyéndose la Alianza para el Progreso, situación que genera la elaboración de los planes de desarrollo, como la búsqueda de recursos externos para su ejecución.

De manera individual y como parte de ese movimiento, los ministros de Salud de la Región establecen unidades de planificación y elaboran planes nacionales de salud, generalmente como parte de los planes nacionales de desarrollo. Emerge en ese momento la prominencia del Centro Panamericano de Planificación y, además, se difunde y aplica, en apoyo a los esfuerzos nacionales, el método CENDES/OPS (método de Planificación diseñado por el Centro de Estudios del Desarrollo - CENDES - de la Universidad Central de Venezuela en años 60-70, y que fue difundido por la OPS en toda la región). De manera global se aprecia la importancia de los esfuerzos conjuntos y mancumunados y se impulsa la adopción de Planes Regionales de Salud para las Américas, los cuales debían seguir los lineamientos de la Carta de Punta del Este, suscrita por los países del continente, como del Plan Decenal de Salud para las Américas.

En Venezuela se da para ese momento un pacto político de gobernabilidad entre los tres principales partidos, por medio del cual se comprometen a respetar los resultados de la elecciones y a participar en la gestión de gobierno. Este pacto denominado Punto Fijo, por ser el nombre de la casa donde se llevó a cabo la reunión que lo hizo posible, permitió elaborar una nueva Constitución de la República en 1961, la cual recoge, entre sus componentes estratégicos, la declaración de la salud como un derecho gratuito de todos los venezolanos.

\section{Las ciencias sociales en el aparato del Estado}

Este fue un momento oportuno para algunas definiciones de políticas a propósito de este marco constitucional y los derechos de la salud. Se estableció la salud como un aspecto destacado de los planes generales de desarrollo, vinculado a programas de Desarrollo Rural Integrado y los Planes $\mathrm{N}$ acionales de Alimentación y Nutrición entre los más importantes. La inversión en salud en este periodo fue notable, con grandes esfuerzos en la planificación, logrando cambios significativos en la cobertura de los servicios de salud, con una orientación hacia el reforzamiento de los aspectos curativos.

Las políticas sociales y de salud en esta década, con tendencia al privilegio de lo curativo sobre lo preventivo, generó automáticamente una formulación de presupuestos-programas concentrados en los gastos de infraestructura hospitalaria, incremento de camas. Situación que influye negativamente en la orientación de las políticas de promoción, educación y participación social en el contexto sanitario que era donde tenían más presencia las ciencias sociales.

Las políticas sociales en el sector salud se orientan en este momento, a darle continuidad a la filosofía de educación sanitaria, integrada a la metodología de D esarrollo Integral de la Comunidad desde la visión de atención y educación de la salud a las comunidades de influencia de las U nidades Sanitarias y los Ambulatorios U rbanos y Rurales del país.

A mediados de esta década, van surgiendo de manera tímida algunas experiencias e iniciativas referidas al modelo de Organización y Desarrollo Integral Comunitario, o Participación Popular, el cual presentaba una visión filosófica más amplia de la participación comunitaria en salud, experiencias referenciadas por organismos internacionales en otros países de América Latina y el Caribe, donde ya se estaba abandonando el paradigma del modelo de desarrollo de la comunidad.

Otros espacios de participación de las ciencias sociales en el sector salud durante esta fase fueron los programas que acompañaron al proceso de reforma agraria y que tenían como propósito mejorar las condiciones de vida de los campesinos y quitarle la base de apoyo que podía encontrar el movimiento guerrillero y que, por su naturaleza, requerían de profesionales y conocimientos sociales. 
Dos actividades merecen destacarse: a) los programas de educación ambiental y de construcción de viviendas rurales en la Dirección de M alariología y Saneamiento Ambiental, los cuales por las características del programa ameritaban la participación de profesionales de las ciencias sociales para la planificación, gestión, monitoreo y evaluación de las actividades directamente en campo; b) el Programa de M edicina Simplificada, que consistía en la capacitación a personas de las diferentes comunidades rurales dispersas e indígenas del país, a objeto de ampliar la cobertura en forma organizada y sustentable de la atención en salud a la población.

Con la expansión de estos programas del gobierno, se incrementa desde mediados de los años sesenta el número y diversidad de profesionales de las ciencias sociales en la estructura del Ministerio de Sanidad a nivel central. Sociólogos, psicólogos, educadores, antropólogos ingresan al aparato del Estado con el objeto de ir fortaleciendo los nuevos programas de capacitación en salud, investigación social en salud y desarrollo comunal. Por ese mismo tiempo, en 1964, se introduce al primer cientista social dentro del grupo de docentes para formar estudiantes en la Escuela de Salud Pública. A partir de esa fecha comienza una progresiva incorporación de sociólogos y antropólogos en las escuelas de medicina del país (M achado, 1970).

Este proceso fue facilitado y forzado por la creciente graduación de profesionales de estas áreas que comienzan a graduarse desde inicios de los años sesenta. Las escuelas universitarias de ciencias sociales habían iniciado sus actividades a mediados de los años cincuenta: la Escuela de Sociología y Antropología y la Escuela de Psicología de la Universidad Central de Venezuela se crearon en 1956, y la Escuela de Ciencias Sociales de la Universidad Católica Andrés Bello en 1959, por lo tanto luego de cuatro años de estudio, que era la duración de las carreras para ese momento, salieron al mercado de trabajo profesionales que pudieron ingresar a la burocracia del Estado en expansión.

Se puede enmarcar la direccionalidad de los esfuerzos de las ciencias sociales en el sector salud en este periodo como una fase de transición filosófica y conceptual del modelo de la educación sanitaria, por el modelo de educación para la salud. Este nuevo modelo viene a facilitar una estructura conceptual y metodológica más amplia en la comprensión integral de los proce sos de salud. I gualmente, lo referente a la superación de los principios de desarrollo de la co- munidad, como una expresión utilitaria o colaborativa de la población en las actividades dirigidas por los servicios de salud. Y de esta forma viabilizar otras alternativas metodológicas en el trabajo comunitario con premisas de participación ciudadana más crítica y constructiva.

\section{Las ciencias sociales en la academia}

Si bien desde mediados de los años cincuenta se fomenta la incorporación de las ciencias sociales en el campo de la medicina de manera sistemática, es a partir de los años 60 que los Departamentos de M edicina Preventiva y Social de las universidades nacionales se incorporan a la formación de recursos humanos en salud pública. En un principio la enseñanza de disciplinas propias de las ciencias sociales estaba concentrada en un nivel más especializado, es decir, en los postgrados médicos. A partir de 1960 con la celebración de el Primer Seminario Nacional de Educación M édica, se crearon las bases para la creación de los Departamentos de M edicina Preventiva y Social y se comenzaron a introducir estas materias en el área de pregrado, visualizán dolos como parte de la formación básica del médico general. Anterior a su creación sólo existían programas docentes 0 cátedras las cuales en ocasiones llevaron el mismo nombre, o parecidos, como en la Escuela de M edicina José M aria Vargas, en Caracas, donde existía una materia llamada "socioantropología de la medicina", que dictaba un médico nutricionista y que resultaba muy divertida para los estudiantes por tratarse de relatos de experiencias y vivencias de la relación social que existe entre el médico y los pacientes. Luego se dio de la incorporación de la medicina social a las ciencias sociales como una asignatura con existencia propia dentro del departamento y los contenidos eran una suerte de sociología general y que tenía algunos temas tales como "el médico como líder y agente de cambio" (M achado, 1970; 2002).

En el cuadro 1 puede observarse el proceso de creación de las asignaturas de la ciencias sociales y de los departamentos de M edicina Preventiva y Social en la Escuela de M edicina de la Universidades Publicas del país, que son todas pues en Venezuela no hay escuelas de medicina en universidades privadas.

La enseñanza de la ciencias sociales, en las escuelas de medicina, en principio, estuvo bajo marcos de referencia norteamericanos, con inclinación conductual lo cual le acreditó el nom- 
Cuadro 1

Años de creación de Departamentos de M edicina Preventiva y Social y de la asignatura de ciencias sociales previa al departamento, en distintas escuelas de medicina del país.

\begin{tabular}{lcc}
\hline Escuelas & Año de creación del Departamento & $\begin{array}{c}\text { Año de creación de la asignatura } \\
\text { en Ciencias Sociales }\end{array}$ \\
\hline Carabobo & 1964 & 1960 \\
Zulia & 1963 & 1960 \\
Vargas & 1961 & 1962 \\
LosAndes & 1960 & 1963 \\
Cedes & 1965 & 1963 \\
Oriente & 1964 & 1964 \\
Ciudad Universitaria & 1960 & 1966
\end{tabular}

Fuente: M achado, Yolanda (1970). La enseñanza de las ciencias sociales en los estudios médicos.

bre de Ciencias de la Conducta, donde tuvieron cabida principalmente la psicología, la sociología y la antropología. Para esa época los estudios sociológicos estaban fundamentados en el funcionalismo, los psicológicos en el conductismo y los antropológicos eran esencialmente culturalistas con énfasis en la etnomedicina (Castellanos, 1986). Esta visión del aporte de las ciencias sociales a la salud se reproduce dentro del mundo de la investigación, donde lo social más que destacarse por sus aportes a la comprensión del proceso salud-enfermedad es visto como una consecuencia de lo biológico (Díaz Polanco, 1993). Ejemplo de ello es el modelo de Historia $N$ atural de la Enfermedad (articulado con la teoría sobre el sistema social de T. Parson) caracterizado por una lógica biológica, el cual si bien es considerado de corte reduccionista, algunos actores de ese proceso sostienen que es necesario reconocer que, en buena medida, la integración de las ciencias sociales en las ciencias médicas se le debe a este modelo ( Díaz Polanco, 2002).

M uchas dificultades se encontraron para la incorporación de las ciencias sociales a los estudios médicos: hubo un crecimiento rápido y poco armónico del cuerpo teórico que permitía sustentar la sociología de la medicina; hubo dificultad para impartir conocimientos básicos y generales de las ciencias sociales a personas formadas en un área distinta y con intereses a veces muy distantes de lo social; y hubo resistencia de algunos médicos ante la poca definición de los objetivos y aportes de aspectos sociales al profesional médico (M achado, 1970).

El mundo médico tendió a subestimar los aportes de estas disciplinas en el proceso salud-enfermedad, y este sentimiento lo traslada- ron los estudiantes de medicina quienes las catalogaban como una pérdida de tiempo (Díaz Polanco, 1993). Comentarios como el siguiente muestran menosprecio de las ciencias sociales dentro de la medicina:

D ebe preocupar que en algunas escuelas, el desarrollo dela Psiquiatría, así como el de la Medicina Preventiva y Social, puede haberse hipertrofiado, en detrimento de la preparación científica del estudiante. Me refiero a sus conocimientos de las Ciencias Básicas en sí y a su aplicación a la clínica y a la terapéutica. (...) Esto es importante de tener en cuenta porque en medicina existen muchas situaciones en que lo social, si bien nunca deja de tener importancia, en un momento determinado no constituye el hecho fundamental que el médico debe atender, pues lo curativo - que requiere una preparación determinada pera lograr el éxito - es lo que entonces realmente importa y a menudo con carácter de emergencia (Benaim H, 1969. Citado por M achado $Y, 1970)$.

El marco referencial y el contenido programático utilizado para la enseñanza de las ciencias sociales, por ejemplo, en la Escuela de M edicina J. M. Vargas, para el año electivo 19681969, aprobado en 1967 por el Departamento de M edicina Preventiva y Social, se dijo que era muy sociológico y muy general. Para el año 1969-1970 se modifica el programa de estudios. Se parte de otro marco ya no sociológico sino médico: Historia Natural de la Enfermedad. Este modelo fue adoptado luego del primer curso-seminario sobre ciencias de la conducta aplicadas a M edicina, dictado por la OPS a cargo del Dr. Juan Cesar García y el Dr. Claudio Jimeno, en la Escuela de M edicina del Vargas en 1969. 
A principios de los setenta con la introducción de marxismo, el programa sufre cambios. Los estudiantes eran introducidos en los conceptos básicos del funcionalismo, luego sobre el marxismo y por último se les daban las herramientas para vincularlo con los problemas de salud. Según M achado (2002), esto dificultó más la enseñanza, pues se consideraba un programa exigente para estudiantes que no provenían de las ciencias sociales y con poca motivación hacia la misma. Las asignaturas relativas a las ciencias sociales eran vistas como algo pajizo, de relleno, ante unos alumnos que se caracterizaban por querer ver enfermos, no individuos sanos.

Años después se revisa el programa, se integran todas las asignaturas de la cátedra de medicina preventiva y social y se le cambia el nombre por el cátedra de salud pública, visualizando la salud como un problema colectivo. Se comenzaba por enseñarles estadística en el primer año, seguida de sociología médica, epidemiología, administración sanitaria y al final un internado médico rotatorio, en medios rurales, bajo la supervisión de personal de la cátedra. Cómo método para facilitar la enseñanza se el egían pequeños casos de salud y se llevaban paralelos a las asignaturas. El principal aporte de las ciencias sociales según M achado (2002) en este tiempo fue que permitió una comprensión más amplia del proceso salud-enfermedad, dándole a los médicos una mayor posibilidad de manipular el problema con el cual se enfrentaban.

Uno de los obstáculos que hubo para la buena enseñanza de las ciencias sociales fue la carencia de estudios de postgrado en sociología y/ o antropología de la medicina, la mayoría de los profesores que se incorporaban a dictar estas materias en las escuelas de medicina no tenían formación especializada en esta área ni cursos de postgrado en general, y a muchos no les interesaba el área específica de trabajo y se dedicaban a enseñar sociología teórica o política, 0 a hacer trabajos de investigación que poco o nada tenían que ver con la salud, la enfermedad 0 la medicina. A esta carencia de formación o de interés se le agregaba la insuficiencia de publicaciones que en el área de ciencias sociales y medicina tenían las bibliotecas de las escuelas de medicina o de ciencias sociales y que no ayudaban a estimular el campo de conocimiento

Para los años sesenta solo existía un curso de pregrado conducido por un médico en la escuela de sociología y antropología en la U niversidad Central de Venezuela sobre historia de la medicina en Venezuela, donde se impartían conocimientos relacionados a la sociología médica ( $M$ achado, 1970). Es en los inicios de los años setenta, cuando se transforma el pensum de estudios de la Escuela de Sociología, cuando se abre una opción para realizar tesis de grado en sociología de la medicina bajo la dirección de Jorge Diaz Polanco y Gloria M arrero. A nivel de especializaciones para la época solo se disponía de cursos dictados en la Escuela de Salud Pública (Planificación de la Salud, Clínicas Sanitarias) y la M aestría en Salud Pública.

Para Castellanos (1986) la incorporación de las ciencias sociales en las escuelas de medicina de las universidades se ha caracterizado por tener: un carácter marginal en el conjunto del curriculum; un desempeño académico de tendencia teoricista, poco vinculado con las actividades de terreno o prácticas profesionales de los estudiantes; y un exiguo prestigio entre los estudiantes y el resto de los profesores.

En esta fase sucede un proceso contrario de lo que había ocurrido en la primera, es decir, ya no es el mundo médico que va hacia lo social, sino lo social que se dirige e incorpora hacia la medicina.

\section{Fase IV - Lo social como crítica al poder (1970-1990)}

En los primeros años de la década de los setenta, el marxismo irrumpe en el mundo intelectual latinoamericano y del mismo modo en las distintas escuel as de medicina. La revuelta estudiantil del 68 en Francia y en USA tuvo su eco en Venezuela; dos años más tarde, se llevó a cabo la renovación universitaria por un grupo de estudiantes y profesores disidentes del sistema de enseñanza tradicional y opuestos al dominio que sobre las universidades ejercía un partido comunista que actuaba como si fuese un consulado de la embajada soviética. El movimiento contestatario que se desarrolló en Venezuela tuvo mucha fuerza pues se opuso tanto al capitalismo como al comunismo pro-soviético que gobernaba las universidades públicas.

A partir de allí se introduce entonces la nueva versión del marxismo que venía propiciando el estructuralismo marxista francés de L. Althusser, y que tenía sus versiones en la ciencias políticas en N. Poulantzas y en la sociología urbana en M. Castells. Con el triunfo de S. Allende en Chile se logra generar una difusión de muchos estos materiales y en particular del manual elaborado por M. H arnecker que comien- 
za a ser utilizado en la enseñanza tanto en las escuelas de sociología como en las de medicina. La ciencia social comenzó a ser sinónimo del marxismo en general y de esta corriente en particular.

En este proceso, nuevamente el papel de la OPS es relevante al establecer un nuevo modelo de enseñanza elaborado sobre la base de los principios marxistas. En el año 1972, se realizó en Cuenca, Ecuador, una reunión sobre la Enseñanza de las Ciencias Sociales en Las Facultades de Ciencias de la Salud, en la que participaron representantes de distintos países de la región. En la reunión se hizo un análisis de los modelos teóricos existentes y usados comúnmente en la vinculación de las ciencias sociales y la medicina, de sus consecuencias en ambos campos. Las críticas se centraron en el funcionalismo, modelo imperante para la época, el cual se concibió como un marco con "una concepción estática de los problemas de salud" (OPS, 1974) dando paso a un marco teórico alternativo que era el marxismo. Si bien dentro del terreno de los médicos no eran muy bien vistas las ciencias sociales, con la entrada del marxismo se gana la simpatía de al gunos médicos, quienes por razones personales, tenían una postura ideológica de izquierda. Algunos autores (Castellanos, 1986) consideran que esta reunión contribuyó al fortalecimiento de las ciencias sociales en las distintas facultades de medicina, odontología, entre otras, especialmente en el departamento de medicina preventiva y social, al impulsar investigaciones en el área salud donde las ciencias sociales jugaron un papel determinante desde la elección del problema hasta el análisis e interpretación de resultados.

En la década de los setenta predominaron las investigaciones en trabajo y salud, el cual tiene las versiones de medicina ocupacional y que intentaba mostrar como la explotación del trabajo por el capital dañaba también a los trabajadores quienes eran despojados no solo de la plusvalía sino también de su salud. Luego a finales de la década toma importancia el tema de políticas de salud, inserto dentro del interés por el estudio de los cambios en el Estado y de sus repercusiones en la conflictividad social.

A comienzo de los ochenta se perfila una orientación hacia una visión integral del proceso salud-enfermedad y de las políticas y planes de salud, evidenciada a través de los siguientes indicadores: se retoma la antropología con un enfoque menos culturalista y más integral del hombre en relación con sus perfiles de salud, ejemplo de ello es la apertura en 1984 de un postgrado en antropología médica, por parte de la Escuela de Antropología de la U niversidad Central de Venezuela; el desarrollo de una tendencia más social del conductismo; el replanteamiento de una ecología menos biologicista e inmersa en la conflictividad social; revalorización de la genética y la inmunología con una visión más integral entre lo biológico y lo social en los fenómenos humanos; recuperación en la sociología aplicada a la salud de la categoría "poder" como elemento central de análisis del conflicto social (Castellanos, 1986).

La continuidad de la visión marxista estuvo dada por la influencia de M. Foucault y sus ensayos sobre la micro-física del poder (Foucault, 1979). La crítica al poder que en la influencia marxista tenía una expresión política y de lucha de clases, adquiere una dimensión distinta con Foucault, pues se vuelve cultural, microsocial y cotidiana. Ya no se trata de las corporaciones, de las acciones de los empresarios o terratenientes, sino de los sutiles vínculos que relacionan al médico con el paciente, al hospital con la cárcel a través del panóptico. En este caso y de manera distinta se regresa al estudio del sistema médico y se abandona el de las condiciones sociales que producen la salud y la enfermedad, que era más propio del marxismo, pero ambas corrientes se unifican en su espíritu crítico y contestario del poder en todas sus formas, en la empresa, en los aparatos del Estado, en la palabra.

Lo que es muy particular de este proceso en Venezuela es que la difusión del marxismo en la academia ocurre en el mismo período que se da una alza importante en los precios del petróleo y el ingreso petrolero se triplica de un año para otro llegando al país una aval ancha de dinero que constituyó la época de mayor bonanza financiera de la nación. En ese contesto las prédicas revolucionarias tenían muy poca acogida entre la población que veía crecer su ingreso y, que sus posibilidades de satisfacer sus expectativas de consumo no solo eran reales, sino que, en momentos, seveían excedidas por tener más dinero que objetos que comprar.

A inicios de los setenta (1971) por ley de presupuesto del M inisterio de Sanidad se cambia la nomenclatura de la Dirección de Asuntos Sociales por Dirección de Bienestar Social, pero es en 1974, el año en que llegan los inmensos recursos petroleros al gobierno, cuando se crea en la dirección una estructura para la planificación, programación y dotación de los M ódu- 
los de Salud y Bienestar. Este nuevo programa permite el reclutamiento de muchos profesionales de las ciencias sociales, los cuales cumplen un papel de promoción, educación y participación comunitaria en los ambulatorios urbanos de los diferentes estados del país.

En 1979, se inicia un nuevo período de reestructuración fundamentado en una revisión y redefinición de la misión, objetivos y estrategias de la Dirección de Bienestar Social para convertirla en la Dirección de Promoción Social para la Salud. Allí se analizan las amenazas y oportunidades del entorno sociopolítico de Venezuela para ese período, y se diseñan metodologías de promoción, educación con criterios de sustentabilidad de organización y participación comunitaria, en la perspectiva de construir los espacios oportunos para cristalizar el proceso de autogestión en salud. Este compromiso del equipo de las ciencias sociales, generó una planificación integral de actividades desde el desarrollo de la investigación acción-participativa, procesos de capacitación a los equipos locales y a las comunidades en una perspectiva crítica y reflexiva del conocimiento y sus niveles de aplicabilidad de acuerdo a la determinación y priorización de situaciones/problemas específicos; y el desarrollo de estrategias de promoción y educación en correspondencia a los perfiles socio-culturales de cada población.

El resultado de esta combinación de factores fue una suerte de conflicto en los profesionales de las ciencias sociales, pues estaban siendo entrenados con una orientación marxista y crítica, que presumía explotación y penuria, pero se incorporaban a trabajar en un gobierno y una sociedad que tenía abundantes recursos y que le demandaba actuaciones microsociales que permitieran administrar la riqueza para las cuales no estaban preparados ni técnica ni ideológicamente.

\section{Fase V - La diversidad de lo social: de la medicina a la salud (1980 en adelante)}

La riqueza fue tan grande como efímera y la gran cantidad de recursos hicieron crisis a inicios de los años ochenta, coincidiendo con la recesión y decrecimiento que sufre la economía latinoamericana durante esa década. En Venezuela los más altos ingresos per capita se obtuvieron en 1979, y después de 1980 sufre una caída permanente que no se ha detenido veintidós años después. Los años ochenta representan un cambio importante en el país que no logra cómo reaccionar y cómo manejar el conflicto social cuando ya no tiene abundantes ingresos petroleros.

La Organización M undial de la Salud identifica esta década como un momento de transición entre los postulados de Alma-A ta y la Reforma. La evaluación de los resultados de la estrategia de la Atención Primaria en Salud mostró un abanico de posiciones, que iban desde la comprensión de la APS, como un componente del primer nivel dela atención médica del sistema de salud, hasta la ubicación en la estructura funcional del sistema con articulación de una organización comunitaria. A mediados de esta década, parte del seguimiento de estas orientaciones surge como elemento de fortalecimiento de la estrategia de los Sistemas Locales de Salud (SILOS), presentada como una respuesta ante los principios de la descentralización y la iniciativa de los M unicipios Saludables, cuyo objetivo era vincularse a las iniciativas anteriores con énfasis en la educación, promoción y atención en salud.

En el año de 1987 se promulgó la Ley Orgánica del Sistema Nacional de Salud que se proponía la creación de un sistema nacional de salud. En ese contexto las políticas de participación social en el sector salud - a nivel del discurso - tienen mayor relevancia, inclusive sereformula la denominación de una Dirección de Línea a una Dirección Sectorial de Promoción Social para la Salud, posición organizacional que favorece su ubicación estratégica en la toma de decisiones a nivel ministerial. Este cambio de la Dirección de Promoción Social para la Salud condujo nuevamente a evaluar su organización interna en correspondencia a las demandas del sector salud, como al nivel de necesidades y demandas de las poblaciones potenciales beneficiarias del programa y confirmó la promoción de la salud, como una estrategia para el logro de una transformación de la salud de la población, teniendo como base de sustentabilidad la organización y participación de las comunidades. Todo lo cual permitió el fortalecimiento de los equipos locales de las ciencias sociales en las áreas de investigación en salud, capacitación en servicio, promoción, educación para la salud sustentado en la organización y participación comunitaria.

Durante los años 97 y 98 se inició una reestructuración del M inisterio de Sanidad y Asistencia Social, y se cursó ante el Congreso de la República los siguientes proyectos: La Ley Ge- 
neral de Salud, con carácter orgánico, de forma que pueda ordenar otros estamentos legales vigentes; Ley M arco de Seguridad Social; Ley del Subsistema de Salud.

La gestión de salud para ese momento consideró que el viejo modelo de la gestión de salud había colapsado, al no poder responder con eficiencia y eficacia a las turbulencias generadas en su entorno. Se orientaron esfuerzos en el proceso de la descentralización de los servicios de salud.

Solo con la participación activa y consciente de los integrantes de la comunidad organizada y la correcta orientación y ayuda por parte del equipo de salud, se puede conseguir el estado de salud (M inisterio de Sanidad y Asistencia Social, 1997).

Para el año de 1999, se inició una nueva gestión gubernamental la cual fue acompañada por un proceso de cambio de políticas públicas, derivándose la Constitución Bolivariana de Venezuela. La misma demanda un cambio de paradigma de los aspectos sociales y de la salud, estableciéndose como objetivo supremo de la sociedad venezolana alcanzar una justicia social de garantía universal de derechos y de equidad, en el marco de un Estado democrático, descentralizado, participativo y social de derecho y de justicia que defiende como valores superiores la vida, la libertad, la igualdad, la solidaridad la democracia y la responsabilidad social en general (M inisterio de Salud y Desarrollo Social, 2001). Esta situación produce cambios en el sector salud desde el cambio de la denominación del M inisterio de Sanidad y Asistencia Social por M inisterio de Salud y Desarrollo Social.

Las premisas que sustentan esta nueva visión de salud del país se expresa en: Asumimos la salud y el desarrollo social como derecho de los ciudadanos y ciudadanas, y su garantía como responsabilidad del Estado. Para ello nuestros esfuerzos están dirigidos a mejorar la calidad de vida; que la prestación de nuestros servicios sea eficiente y privilegie a los que por muchos años han sido excluidos, para alcanzar el equilibrio social (M inisterio de Salud y Desarrollo Social, 2001)

La forma de operacionalizar estas premisas, según los documentos del Ministerio de Salud, se basó en el modelo de atención integral en salud y desde la red de ambulatorios, cuyo efecto transformador integral de la vida local y regional lo convierten en una estrategia para la política del desarrollo social.

Este escenario conceptual lleno de ideas transformadoras de la justicia social como mar- co a la visión de la salud como valor en la sociedad actual venezolana, paradójicamente, no guarda relación con el proceso de reestructuración del Ministerio de Salud y Desarrollo Social, donde cambió la denominación de Asistencia Social por Desarrollo Social, pues en Iugar de potenciar el espacio de lo social, construido con el effuerzo sistemático de profesionales de las ciencias sociales desde el año 1936, quedó eliminada en la estructura funcional del ministerio por un decreto.

Con la desaparición de esta unidad programática y de un equipo transdiciplinario de las ciencias sociales formado en varios periodos, se perdió un espacio de organización y de formulación de políticas sociales en el marco de la salud de un país, que hoy más que nunca amerita de la articulación de ideas y experiencias para diseñar líneas estratégicas de salud colectiva.

En las universidades el proceso significa una mayor consolidación de los grupos de investigación y una mayor presencia de las ciencias sociales. En la Escuela de Salud Pública de la Universidad Central de Venezuela se plantean como postulados de sus políticas educativas: que los factores socioculturales son determinantes básicos en la distribución de la enfermedad, juegan un papel importante en la etiología de la enfermedad, definen que condiciones deben ser consideradas como problemas de salud pública y las actividades que pudieran ser realizadas en torno a la solución de ellos y determinan las respuestas del individuo, del grupo y de la comunidad frente a los problemas y programas de salud pública.

Esta fase implica al mismo tiempo una mayor variedad en los abordajes teóricos y metodológicos; se puede decir que es pluriparadigmática, porque coexisten diversos modos de investigar, enseñar e intervenir con las ciencias sociales en el campo de la salud y la enfermedad. La caída del muro de Berlín desconcierta a los más aferrados a la visión marxista y los obliga a replantearse sus mismos ideales con otros modos de alcanzarlos. Por otra parte en este período empiezan a entrar los organismos multilaterales, en especial el Banco M undial y Banco Interamericano de Desarrollo, como actores fundamentales en la definición de las políticas sociales $y$, entre ellas de manera privilegiada, las políticas de salud.

Otro factor importante de estos cambios fue la creación del Programa Especial de Investigación y Formación en Enfermedades Tropicales (TDR), el cual empieza a tener una impor- 
tante presencia en la investigación social de dichas enfermedades, pues no tiene las restricciones de la OM S-OPS que deben canalizar todas las actividades a través del gobierno, sino que pueden aproximarse directamente a los grupos de investigación de las universidades. Estas investigaciones estaban claramente orientadas al estudio de las condiciones sociales y ambientales que favorecían la transmisión o la prevención y el control de estas enfermedades transmitidas por vectores, por lo tanto exigían unas herramientas metodológicas y conceptuales apropiadas a este tema de investigación y que obligaba a alejarse tanto de las perspectivas macro-sociales como de las críticas de la medicina, se volvía a trabajar en el proceso de saludenfermedad, no del poder médico.

Paralelamente y por la presión del "consenso de Washington" se procede a realizar un conjunto de cambios en el Estado venezolano, y uno de los más importantes fue el proceso de descentralización del sistema de salud, dejando al nivel central la función de rectoría, pero transfiriendo a los estados la ejecución de los programas. Con este cambio se fueron creando instancias locales de salud, como fundaciones de Estado que permitía un control más cercano de las actividades y que en al gunos casos conllevó a una mayor presencia de las ciencias sociales en las actividades de salud.

En 1989 se inició en Venezuela un programa regional de Pequeñas Becas para Investigación en aspectos sociales, económicos y culturales de la enfermedades tropicales. Este programa fue diseñado y administrado por el Laboratorio de Ciencias Sociales (LACSO) durante seis años (1989-1996) y financió a más de cien investigadores en toda América Latina. Su impacto fue muy importante en la región y también en Venezuela, pues permitió incorporar a nuevos científicos sociales al área e la salud sin que estuvieran trabajando en escuelas 0 centros de investigación de medicina, y logró crear una comunidad científica (Briceño-León, 1994; Briceño-León y Vlassoff, 2000)

En los años noventa se formalizan distintos grupos de investigación y formación en el área de salud en las distintas universidades. En el CENDES se mantiene un grupo de investigación en políticas de salud liderizado por J. Diaz Polanco y T. M aingo, así como una opción dentro de la maestría y doctorado en el área de salud. En los años noventa se inicia un grupo que trabaja en Ecosistemas y Salud bajo la Dirección de L. Y ero, la cual establece un curso de maes- tría sobre ese mismo tema y desarrollan estudios sobre la explotación minera y salud.

En la U niversidad de Zulia el equipo de Espacio A bierto trabaja en distintas dimensiones sociales de salud, habiendo desarrollado un trabajo importante con las investigaciones sobre la lepra con Alexis Romero a la cabeza. En el Instituto de Biomedicina, se apertura formalmente la integración del componente social en el contexto del control de las diferentes endemias, entre ellas: Ieishmaniasis, lepra y oncocercosis, realizando intervenciones de promoción de la salud en distintas comunidades, como el desarrollo de líneas de investigaciones operacionales y la capacitación de recursos comunitarios en educación y participación comunitaria.

En la Universidad de Carabobo un grupo de investigación venía trabajando desde los años ochenta en la relación entre pobreza y salud, así como diversas enfermedades metaxénicas: esquistosomiasis y Enfermedad de Chagas, bajo la dirección de F. Bello y Y. Sevilla. Y a fines de los noventa se establece en el curso de doctorado en ciencias sociales una mención en Salud y Sociedad que cursan tanto médicos como profesionales de las ciencias sociales.

Lo que durante un tiempo fue la Escuela de Malariología se transformó recientemente en el Instituto de Altos Estudios en Salud Pública Dr. Arnoldo Gabaldón. En este centro dependiente del M inisterio de Salud hay cursos de maestría en enfermedades transmisibles y en Salud O cupacional que tienen un importante componente social en su plan de estudios.

En los postgrados de psicología social tanto de la Universidad Central como de la Universidad Simón Bolívar, hay grupos de investigación que trabajan en el área de psicología de la salud. El grupo ha adquirido bastante importancia y existe una A sociación Venezolana de Psicología de la Salud que tiene su membresía y realiza congresos nacionales.

El grupo de antropólogos que trabaja en el área de la salud se mueve en distintas instancias, hay unos grupos tanto en la U niversidad Central de Venezuela, como el Instituto Venezolano de Investigaciones Científicas (IVIC), dedicados a la antropología física que trabaja con nutrición y antropometría, otro grupo se dedica a estudiar las condiciones de salud de las poblaciones indígenas, y un tercer grupo concentrado en la U niversidad de los Andes, dirigido por J. Clarac, que realiza estudios de etnomedicina.

En 1994 se realizó en Caracas el encuentro fundacional de la sección latinoamericana del 
International Forum for Social Sciences and $\mathrm{H}$ ealth (IFSSH), el cual permitió hacer un balance del Estado de arte de esta área del conocimiento y cuyas deliberaciones fueron publicadas en el libro Las ciencias y salud en América Latina: un balance (Briceño-León, 1999). El Laboratorio de Ciencias Sociales - LACSO - actuó como sede del Secretariado Latinoamericano entre 1994 y 1998 y como secretariado mundial del IFSSH desde 1997 hasta el año 2002.

De igual modo en los años noventa se formó la Asociación Venezolana de Sociología y Salud (AVESSA) que ha organizado tres congresos: dos Congresos Venezolanos de Ciencias Sociales y Salud, el primero en 1996 y el segundo en el 2002 y, el V Congreso Latinoamericano de Ciencias Sociales y M edicina, en Isla M argarita en 1999 el cual surgió el libro Salud y equidad: una mirada desde las ciencias Sociales (BriceñoLeón et al., 2001).

En esta fase como puede observarse se logra una maduración de la comunidad de las ciencias sociales y la salud y permite una diversidad en los enfoques y en los temas y una pluralidad metodológica e ideológica que se ve reflejada tanto en los temas de las investigaciones como en las orientaciones de los cursos de postgrado. Para algunos esa diversidad pudiera constituir una debilidad, a nosotros nos parece que es una muestra de fortaleza y de creatividad en esta línea de investigación y práctica profesional, pues se hacen estudios macro sociales ligados a la reforma y la política de salud, pero también estudios de comunidades o de la familia y la percepción de los individuos del proceso salud enfermedad, sin que exista una corriente que puede establecerse como la dominante, pero con un espíritu de cooperación e intercambio muy valioso para el crecimiento de la comunidad científica.

\section{Conclusiones}

Como se habrá podido observar de la descripción antes realizada, el papel de las ciencias so- ciales en la medicina y la salud en Venezuela ha ido cambiando con las transformaciones que han ocurrido en la sociedad, el Estado y la academia. De al guna manera las ciencias sociales han contribuido a la modernización de la sociedad venezolana y han sido al mismo tiempo víctimas de esas mismas mutaciones. Venezuela, como muchas otras sociedades latinoamericanas, tiene una modernidad inconclusa (Briceño-León, 1999) que se expresa en las paradojas de la organización del aparato productivo y del Estado, pero también en la situación epidemiológica que reúne en un mismo territorio indicadores de la morbilidad y mortalidad del desarrollo y del atraso, y un sistema de salud que oscila entre la sofisticada tecnología y las carencias de los insumos más el ementales, entre la abundancia y la miseria.

La práctica de las ciencias sociales que se ocupan de la salud no están exentas de las grandezas y miserias que ocurren en la sociedad en la cual trabajan. Quizá por motivos de la propia formación profesional se puede tener un poco más de conciencia sobre lo que ocurre, pero, las limitaciones como actores son las mismas que padecen los otros ciudadanos.

Por eso es que se tiene la doble obligación de ser un crítico de la sociedad y el sistema de salud, y, al mismo tiempo, de contribuir activamente con sus cambios y transformaciones. No es posible quedarse solo en la crítica, hay también que dar salidas y soluciones, aunque siempre sean transitorias e imperfectas.

Las ciencias sociales tienen hoy un lugar bien ganado en la salud y la medicina de Venezuela, por su presencia y contribución en los programas del Estado, por el desarrollo de investigaciones que han aportado conocimientos útiles para las acciones de salud, por su organización asociativa y por su labor crítica ante el poder y su enmascaramientos en múltiples manifestaciones. Quizá el reto mayor ahora es poder cubrir con todas las expectativas que las ciencias sociales han creado en la propia sociedad y contribuir eficiente al verdadero cambio social que signifique la modernización de Venezuela. 


\section{Referencias bibliográficas}

Briceño-León R 1994. Setting up a scientific community by means of a small grants program: the Latin American experience. Acta Tropica 57:201-209.

Briceño-León R 1999. La sociedad del siglo XX: una modernidad inconclusa. Ponencia presentada en el Seminario Visiones del Siglo XX Venezolano, con motivo del $V$ Centenario de Venezuela. Caracas.

Briceño-León R \& Vlassof 2000. La experiencia de construcción de una comunidad científica en ciencias sociales y enfermedades tropicales en América Latina, pp. 11-24. In R Barrada \& R Briceño-León (orgs.). Doenças endêmicas. Fiocruz, Rio de Janeiro.

Briceño-León, M inayo M C \& Coimbra C (coords.) 2001. Salud y equidad: una mirada desde las ciencias sociales. Fiocruz, Rio de Janeiro, 384pp.

Castellanos PL 1986. Las ciencias sociales en salud en Venezuela, pp. 143-161. In E Duarte Nunes (ed.). Ciencias sociales y salud en América Latina tendencias y perspectivas. OPS - CIESU

Díaz Polanco J 1993. La investigación sobre salud en Venezuela: el papel de las ciencias sociales. Cuadernos del CENDES 24(2):71-95.

Díaz Polanco J 2002. Contribución de las ciencias sociales a la enseñanza de la salud pública. Ponencia presentada en el XV Aniversario del Instituto $\mathrm{N}$ acional de Salud Pública de M éxico. Cuernavaca.

Foucault M 1973. Naissance de la clinique: archeologie de la regard medicale. Press U niversitaires de France, París.
Foucault M 1979. M icrofísica del poder. Editorial de la Piqueta, M adrid.

H osbawn E 1995. H istoria del siglo XX. Editorial Grijalbo, Barcelona.

M achado Y 2002. Entrevista realizada en persona a la Soc. Yolanda M achado, el 6 de marzo del 2002. Caracas.

$M$ achado Y 1970. La enseñanza de las ciencias sociales en los estudios médicos. Evaluación y análisis comparativo de dos sistemas docentes implementados en la Escuela de M edicina José M aría Vargas. Trabajo de ascenso correspondiente a la categoría de profesor asistente, Facultad de M edicina. Universidad Central de Venezuela, Caracas, 134pp.

M inisterio de Salud y Desarrollo Social 2001. Plan de Salud 2001. Caracas.

M inisterio de Salud y Desarrollo Social 2001. Plan Estratégico Social 2001-2001. Caracas.

M inisterio de Sanidad y Asistencia Social 1997. Reestructuración del M inisterio de Sanidad y Asistencia Social. Caracas.

Organización Panamericana de la Salud 1974. Aspectos teóricos de las ciencias sociales aplicadas a la medicina. Reimpreso de Educación M édica y Salud 4(8):354359

Artigo apresentado em 19/9/2002

A provado em 30/9/2002

Versão final apresentada em 10/10/2002 